\title{
Sitagliptin inhibits endothelin-1 expression in the aortic endothelium of rats with streptozotocin-induced diabetes by suppressing the nuclear factor-кB/IкB $\alpha$ system through the activation of AMP-activated protein kinase
}

\author{
SONG-TAO TANG ${ }^{1-3}$, HUAN SU $^{2}$, QIU ZHANG ${ }^{3}$, HAI-QIN TANG $^{4}$, CHANG-JIANG WANG $^{3}$, \\ QING ZHOU ${ }^{2}$, WEI WEI ${ }^{1}$, HUA-QING ZHU ${ }^{2}$ and YUAN WANG ${ }^{1,2}$ \\ ${ }^{1}$ Institute of Clinical Pharmacology, ${ }^{2}$ Laboratory of Molecular Biology and Department of Biochemistry, \\ Anhui Medical University, Hefei, Anhui; Departments of ${ }^{3}$ Endocrinology and ${ }^{4}$ Geriatrics, \\ The First Affiliated Hospital of Anhui Medical University, Hefei, Anhui, P.R. China
}

Received April 9, 2015; Accepted April 8, 2016

DOI: $10.3892 /$ ijmm.2016.2578

\begin{abstract}
Emerging evidence suggests that dipeptidyl peptidase-4 (DPP-4) inhibitors, including sitagliptin, exert favourable effects on the vascular endothelium. DPP-4 inhibitors suppress the degradation of glucagon-like peptide-1 (GLP-1), which has been reported to enhance nitric oxide (NO) production. However, the effects of DPP-4 inhibitors on endothelin-1 (ET-1) expression in the aorta, as well as the underlying mechanisms responsible for these effects, have yet to be investigated in animal models of diabetes mellitus (DM). In the present study, the rats were randomly divided into the following four groups: i) control; ii) DM; iii) DM + low-dose sitagliptin (10 mg/kg); and iv) DM + high-dose sitagliptin (30 mg/kg). Apart from the control group, all the rats received a high-fat diet for 8 weeks prior to the induction of diabetes with an intraperitoneal injection of streptozotocin. The treatments were then administered for 12 weeks. The serum levels of ET-1, NO, GLP-1 and insulin were measured as well as endothelial function. The expression of ET-1, AMP-activated protein kinase (AMPK) and nuclear factor $(\mathrm{NF})-\kappa \mathrm{B} / \mathrm{I} \kappa \mathrm{B} \alpha$ were determined. After 12 weeks of treatment, the diabetic rats receiving sitagliptin showed significantly elevated serum levels of GLP-1 and NO, and reduced levels of ET-1. Moreover, sitagliptin significantly attenuated endothelial dysfunction as well as the remodeling of the aortic wall. Notably, sitagliptin inhibited ET-1 expression at the transcriptional and translational level in the aorta, which may have been mediated by the suppression of the $\mathrm{NF}-\kappa \mathrm{B} / \mathrm{I} \kappa \mathrm{B} \alpha$ system induced by
\end{abstract}

Correspondence to: Professor Yuan Wang or Professor Hua-Qing Zhu, Laboratory of Molecular Biology and Department of Biochemistry, Anhui Medical University, 81 Meishan Road, Hefei, Anhui 230032, P.R China

E-mail: aydesm-1@163.com

E-mail: aydzhq@126.com

Key words: sitagliptin, endothelin-1, diabetes, aortic endothelium
AMPK activation. The majority of the above-mentioned effects were dose dependent. Taken together, the findings of the present study indicate that sitagliptin inhibits ET-1 expression in the aortic endothelium by suppressing the $\mathrm{NF}-\kappa \mathrm{B} / \mathrm{I} \kappa \mathrm{B} \alpha$ system through the activation of the AMPK pathway in diabetic rats. These findings further demonstrate some of the vasoprotective properties of DPP-4 inhibitors in vivo.

\section{Introduction}

The prevalence of diabetes mellitus (DM) is expected to rise significantly, and DM is viewed as a public health problem of epidemic proportions (1,2). DM-induced vascular diseases are major contributors to morbidity and mortality in the aging population (3).

The endothelium is composed of a monolayer of cells covering the vascular lumen and it provides a continuous barrier between the elements of blood and the arterial wall. The endothelium plays an important role in the regulation of vascular tone, by balancing the release of endothelium-derived vasodilatory factors, such as nitric oxide (NO) and prostacyclin, with endothelium-derived vasoconstrictory factors including endothelin-1 (ET-1), angiotensin II and reactive oxygen species (ROS) (4,5). In diabetes, the disruption of the balance between vasodilatory and vasoconstrictor actions in the endothelium is considered as a crucial event in the initiation of the atherosclerotic process.

The incretin hormone, glucagon-like peptide-1 (GLP-1), is secreted postprandially from the L-cells of the lower gut and has a short half-life of minutes before it is rapidly degraded by dipeptidyl peptidase-4 (DPP-4) (6). Sitagliptin is a stable, orally effective DPP-4 inhibitor, which increases the active concentration and duration of action of GLP-1 (7,8). GLP-1 plays a decisive role in glucose-stimulated insulin release and the preservation of plasma glucose homeostasis. Notably, GLP-1 has been reported to exert cardioprotective and vasodilatory effects and to attenuate vascular endothelial dysfunction in vivo and in vitro (9-13). Most studies have focused on vasodilatory actions, which are principally mediated by NO, a vasodilator 
and anti-inflammatory molecule produced by the action of endothelial nitric oxide synthase (eNOS) $(14,15)$. However, little is known regarding vasoconstrictor actions on the vascular endothelium which are partly mediated by ET-1, a potent vasoconstrictor and pro-inflammatory molecule secreted by the endothelium in animal models.

Thus, in the present study, we established a rat model of diabetes-associated arteriosclerosis in order to determine whether sitagliptin attenuates endothelial dysfunction and inhibits ET-1 expression in the aortic endothelium and to explore the underlying mechanisms responsible for these effects.

\section{Materials and methods}

Animal experimental procedures. Twenty-five male SpragueDawley rats (6 weeks old, weighing approximately 150-180 g) were obtained from the Experimental Animal Centre of Anhui Medical University (Hefei, China). The rats were randomly divided into the following four groups: i) control group $(n=7)$; ii) DM group ( $\mathrm{n}=6)$; iii) $\mathrm{DM}+$ low-dose sitagliptin $(10 \mathrm{mg} / \mathrm{kg})$ treatment group $(n=6)$; and iv) $D M+$ high-dose sitagliptin $(30 \mathrm{mg} / \mathrm{kg})$ treatment group $(\mathrm{n}=6)$. All groups were subjected to a $12: 12 \mathrm{~h}$ light-dark cycle (lights on at 06:00) under controlled conditions of temperature $\left(22 \pm 1^{\circ} \mathrm{C}\right)$ and humidity (50-60\%). With the exception of the control group, all of the rats received a high-fat (HF) diet (2\% cholesterol, $10 \%$ lard, and $88 \%$ normal diet) and sufficient water for 8 weeks, after which time, they were subjected to an intraperitoneal glucose tolerance test (IPGTT) and an intraperitoneal insulin tolerance test (IPITT). The rats in the DM and the sitagliptin treatment groups were injected once intraperitoneally with a dose of streptozotocin $(25 \mathrm{mg} / \mathrm{kg}$; Sigma-Aldrich, St. Louis, MO, USA) to induce diabetes and blood glucose levels were tested 1 week after the streptozotocin injection. The animals with glucose levels $\geq 11.1 \mathrm{mmol} / 1$ were considered diabetic. The rats in the control and DM groups were given normal saline. The rats in the low-dose $(10 \mathrm{mg} / \mathrm{kg})$ and high-dose $(30 \mathrm{mg} / \mathrm{kg})$ sitagliptin (Merck Serono Co., Ltd., Guangzhou, China) groups received sitagliptin once daily by oral gavage for 12 weeks. The rats were weighed every 3 days and the dosage was adjusted accordingly. All the rats were euthanized at the end of week 21. All experiments were approved by the Ethics Committee of Anhui Medical University (Hefei, China).

Non-invasive, transcutaneous ultrasound measurement of blood flow velocity. Non-invasive, transcutaneous ultrasound evaluation of the blood flow velocity was performed two days prior to the end of the experiment as previously described $(16,17)$. Briefly, the rats were anaesthetized using $10 \%$ chloral hydrate solution and placed in the dorsal decubitus position for the shaving of their abdomens. After $10 \mathrm{~min}$ of rest in the supine position, ultrasonic examination of the abdominal aorta was performed using a 13-MHz ultrasound probe (GES6 two-dimensional Color Doppler Ultrasound Diagnostic Apparatus; GE Healthcare, Piscataway, NJ, USA). The transducer was lubricated with ultrasound gel and placed at the level of the abdominal aorta with minimal pressure. The probe was placed $1.0 \mathrm{~cm}$ precisely below the renal artery to obtain a longitudinal axis view of the abdominal aorta. Image settings were optimized to allow the clearest definition of the abdominal aorta. Images of the abdominal aorta were recorded continuously throughout the entire procedure. The ultrasound probe remained in the same position for the duration of the measurement period. The end-diastolic velocity (EDV) and the peak systolic velocity (PSV) were recorded.

Tissue collection. Following the withdrawal of food overnight, the rats were deeply anesthetized using $10 \%$ chloral hydrate solution $(0.3 \mathrm{ml} / 100 \mathrm{~g}$ body weight). Blood samples were obtained from the abdominal aorta. The aortic arch and the thoracic aorta were removed. The aortic arch was longitudinally cut and fixed in $4 \%$ paraformaldehyde for subsequent pathological and immunohistochemical assays. Part of the thoracic aortas were placed in a dish containing ice-cold Krebs solution (composition in mmol/l: $\mathrm{NaCl}, 120 ; \mathrm{KCl}, 4.7 ; \mathrm{KH}_{2} \mathrm{PO}_{4}, 1.18 ; \mathrm{CaCl}_{2}$, 2.25; $\mathrm{NaHCO}_{3}, 24.5 ; \mathrm{MgSO}_{4} \cdot 7 \mathrm{H}_{2} \mathrm{O}, 1.2$; glucose, 11.1; EDTA, 0.03 ) and continuously aerated with $95 \% \mathrm{O}_{2}$ and $5 \% \mathrm{CO}_{2}$. The samples were then cut into rings ( $3 \mathrm{~mm}$ in length) for the measurement of isometric contractile tension. The remaining thoracic aortas were frozen in liquid nitrogen and stored at $-80^{\circ} \mathrm{C}$ for reverse transcription-quantitative polymerase chain reaction (RT-qPCR) and western blot analysis.

Analysis of markers in blood. Blood samples were taken by cardiac puncture, and the levels of serum fasting blood glucose (FBG), total cholesterol (Tch), low-density lipoprotein cholesterol (LDL-c) and triglyceride (TG) were measured using commercially available spectrophotometric assay kits (Beijing BHKT Clinical Reagent Co., Ltd., Beijing, China). Fasting and postprandial serum GLP-1 levels were measured using an enzyme-linked immunosorbent assay kit (Cusabio, Wuhan, China). Serum levels of ET-1 and insulin were measured using a radioimmunoassay kit (Beifang Biology, Beijing, China). Serum NO levels were measured using a commercial kit (Jiancheng, Nanjing, China). The insulin sensitivity index was calculated using the [1/(FBG $\mathrm{x}$ fasting insulin)] formula (18). All analyses were performed in duplicate.

Hematoxylin and eosin $(H \& E)$ staining. Fixed aortic specimens were dehydrated, embedded in paraffin, sectioned ( $4 \mu \mathrm{m}$ thickness) and stained with H\&E as previously described (19). The artery wall was observed using a microscope (Leica, Wetzlar, Germany) and the mean artery wall thickness was calculated.

Immunohistochemical analysis. Briefly, the sections of the thoracic aorta were deparaffinised, rehydrated, and fixed with methanol- $0.3 \% \mathrm{H}_{2} \mathrm{O}_{2}$ solution for $20 \mathrm{~min}$ at room temperature. The antigens were unmasked by cooling for $30 \mathrm{~min}$ at room temperature after high compression heating. Primary antibodies against ET-1 (Santa Cruz Biotechnology, Inc., Santa Cruz, CA, USA) were added and incubated with the tissue sections overnight followed by sequential incubation with a biotinylated antibody (anti-rat IgG) and horseradish streptavidin for $30 \mathrm{~min}$ at $37^{\circ} \mathrm{C}$. Finally, the samples were incubated with diaminobenzidine for coloration and counterstained with haematoxylin for 2 min to stain the nuclei. Positively-stained cells appeared brown in colour. Images were captured using a microscope (Leica), and the integral optical density values were measured using the JD-801 pathological imaging analysis system (Jiangsu JEDA Science-Technology Development Co., Ltd., Jiangsu, China). 
Measurement of isometric contractile tension. Individual aortic rings were vertically suspended between two stainless steel wire hooks in a jacketed organ bath containing $25 \mathrm{ml}$ Krebs solution. The Krebs solution was replaced at 15-min intervals. The bathing solution was bubbled continuously with a mixture of $95 \% \mathrm{O}_{2}$ and $5 \% \mathrm{CO}_{2}$ at $37^{\circ} \mathrm{C}$. The isometric contractile tension was recorded continuously using a BL-420F biological function experimental system (Chengdu Taimeng Science and Technology Co., Ltd., Chengdu, China). The resting tension applied to the aortic rings was increased incrementally to a final tension of $2 \mathrm{~g}$. The rings were then equilibrated for $45 \mathrm{~min}$. Following equilibration, the rings were precontracted with $1 \mu \mathrm{mol} / 1$ phenylephrine (Phe); once a stable contraction plateau was obtained, either $10^{-8}$ to $10^{-4} \mathrm{~mol} / 1$ acetylcholine (Ach) or $10^{-8}$ to $10^{-4} \mathrm{~mol} / \mathrm{l}$ sodium nitroprusside (SNP) was added to the organ bath cumulatively until a maximal vasodilator response was achieved. Cumulative vasodilator response data are expressed as the percentage of relaxation relative to the Phe-induced precontraction.

$R T-q P C R$. SYBR-Green RT-qPCR was performed in order to detect the mRNA levels of ET-1. Total RNA was extracted from the aortic tissue of rats using TRIzol reagent (Invitrogen, Grand Island, NY, USA). RNA was reverse transcribed to cDNA using the PrimeScript RT reagent kit (Takara Bio, Otsu, Japan). qPCR was performed with PikoReal (Thermo Fisher Scientific, Waltham, MA, USA) using SYBR Premix Ex Taq (Takara Bio). The relative quantification values for the expression of these genes were calculated using the $\Delta \Delta C T$ method and corrected using a housekeeping gene. The following primer sequences for the analysis of ET-1 and GAPDH mRNA were used: ET-1 forward, 5'-CAACCAGACACCGTCCT CTT-3' and reverse, 5'-CTTGGAAAGCCACAAACAGC-3'; and GAPDH forward, 5'-TCATTGACCTCAACTACA-3' and reverse, 5'-CAAAGTTGTCATGGATGACC-3'.

Western blot analysis. The aortas were washed three times in TBS, and then lysed in RIPA buffer. The lysates were centrifuged at $14,000 \mathrm{xg}$ for $30 \mathrm{~min}$ at $4^{\circ} \mathrm{C}$. The total protein concentration of each sample was measured using a Micro BCA Protein Assay reagent kit (Pierce, Rockford, IL, USA). The same amount of lysate from each line in SDS sample buffer was resolved by $10 \%$ SDS-polyacrylamide gel electrophoresis and electroblotted onto a PVDF membrane, which was then blocked with 5\% fat-free milk in TBST (TBS, $0.1 \%$ Tween-20) for $1 \mathrm{~h}$ at room temperature. The ET-1 Ab (1:250 dilution; sc-21625-R; Santa Cruz Biotechnology, Inc.); phosphorylated (p-)AMP-activated protein kinase (AMPK) ( $\mathrm{Thr}^{172}$ ) $\mathrm{Ab}$ (1:500 dilution; no. 2535) and p-nuclear factor (NF) $-\mathrm{\kappa B}$ p65 Ab (1:500 dilution; no. 3033) (both from Cell Signaling Technology, Inc., Danvers, MA, USA); and p-IкB $\alpha$ Ab (1:250 dilution; sc-8404; Santa Cruz Biotechnology, Inc.) were incubated overnight at $4{ }^{\circ} \mathrm{C}$, followed by incubation with secondary antibodies conjugated with horseradish peroxidase (Beijing Zhongshan Jinqiao Biotechnology Co., Ltd., Beijing, China). Specific bands of the target proteins were stained with chemiluminescence reagent (Pierce). Densitometric scanning of the exposed X-ray film was used for semi-quantitative measurement of the protein bands. $\beta$-actin levels were used to normalise the relative protein expression levels.
Statistical analysis. All statistical analyses were performed using SPSS software, version 18.0. All data are presented as the means \pm standard deviation (SD). Comparisons between the groups were conducted using one-way analysis of variance (ANOVA) and the Student-Newman-Keuls (SNK) method. A P-value $<0.05$ was considered to indicate a statistically significant difference.

\section{Results}

IPGTT and IPITT. Following 8 weeks of HF feeding, IPGTT and IPITT results revealed that the blood glucose levels in the rats fed an HF diet were significantly higher than those in the control rats at baseline (IPITT only) and at 15, 30, 60 and 120 -min time-points $(\mathrm{P}<0.05)$. The area under the curve (AUC) for the glucose level increased significantly in the rats fed an HF diet for 8 weeks $(\mathrm{P}<0.05)$ (Fig. 1). These results suggested that the rats fed an HF diet were insulin resistant.

Metabolic parameters in diabetic rats. Statistical analysis showed that the body weight and the blood glucose levels were significantly changed in the DM group, and that sitagliptin reduced the extent of weight loss as well as the high FBG levels during the experimental period (Fig. 2). Following 12 weeks of treatment, FBG, Tch, TG, LDL-c and insulin resistance were significantly higher in the diabetic rats compared with the control group $(\mathrm{P}<0.05)$. The administration of high-dose sitagliptin significantly reduced FBG levels and insulin resistance $(\mathrm{P}<0.05)$. However, the levels of Tch, TG and LDL-c were not significantly affected by low dose of sitagliptin $(\mathrm{P}>0.05)$ (Table I).

Effects of sitagliptin on serum levels of GLP-1, ET-1 and NO. The induction of diabetes significantly increased the serum levels of ET-1 and reduced the levels of fasting and postprandial GLP-1 as well as NO $(\mathrm{P}<0.05)$. The adminstration of high- and low-dose sitagliptin significantly reduced the serum levels of ET-1 and elevated the serum levels of GLP-1 as well as $\mathrm{NO}$ to varying extents $(\mathrm{P}<0.05)($ Fig. 3$)$.

Endothelial function and velocity of blood flow. We then examined the effects of sitagliptin on vascular endothelial function in the diabetic rats. Isometric contractile tension experiments on isolated aortic rings were performed. As illustrated in Fig. 4, in the DM group, we observed inhibition of the cumulative contraction-relaxation response to Ach in the thoracic aortic rings compared with the controls $(\mathrm{P}<0.05)$, whereas there were no significant changes in the cumulative contraction-relaxation response to SNP $(\mathrm{P}>0.05)$. The administration of different doses of sitagliptin partly reversed the diabetes-induced impairment of the cumulative concentration-vasodilator responses to Ach in the thoracic aortic rings precontracted with $\mathrm{Phe}(\mathrm{P}<0.05)$ and had no effect on the SNP-induced cumulative contraction-relaxation responses (Fig. 4C and D). The contractile response to Phe in the untreated diabetic rats was lower than that in the control rats and the rats treated with high-dose sitagliptin (Fig. 4B). In addition, performing non-invasive transcutaneous ultrasound measurement of blood flow velocity in the abdominal aorta showed that PSV was significantly reduced in the DM group, 
Table I. Basic parameters of rats in different experimental groups following 12 weeks of treatment.

\begin{tabular}{lcccc}
\hline & Controls $(\mathrm{n}=7)$ & DM $(\mathrm{n}=6)$ & DM + Sitagliptin 10 $(\mathrm{n}=6)$ & DM + Sitagliptin 30 $(\mathrm{n}=6)$ \\
\hline Weight $(\mathrm{g})$ & $485.00 \pm 17.15^{\mathrm{a}}$ & $377.33 \pm 19.07$ & $457.83 \pm 37.03^{\mathrm{a}}$ & $456.67 \pm 59.54^{\mathrm{a}}$ \\
FBG $(\mathrm{mmol} / \mathrm{l})$ & $4.93 \pm 0.27^{\mathrm{a}}$ & $16.92 \pm 1.79$ & $13.67 \pm 1.61^{\mathrm{a}}$ & $7.1 \pm 2.15^{\mathrm{a}, \mathrm{b}}$ \\
Tch $(\mathrm{mmol} / \mathrm{l})$ & $2.77 \pm 0.14^{\mathrm{a}}$ & $6.58 \pm 1.6$ & $6.56 \pm 1.28$ & $6.28 \pm 1.06$ \\
TG $(\mathrm{mmol} / \mathrm{l})$ & $1.14 \pm 0.07^{\mathrm{a}}$ & $5.01 \pm 1.39$ & $4.71 \pm 1.00$ & $4.48 \pm 0.51^{\mathrm{a}}$ \\
LDL-c (mmol/l) & $1.08 \pm 0.05^{\mathrm{a}}$ & $4.65 \pm 1.5$ & $4.58 \pm 0.97$ & $4.29 \pm 0.75$ \\
Fasting insulin (uIU/ml) & $12.07 \pm 3.36^{\mathrm{a}}$ & $44.95 \pm 11.07$ & $30.68 \pm 7.06^{\mathrm{a}}$ & $16.90 \pm 4.17^{\mathrm{a}, \mathrm{b}}$ \\
lnISI & $-4.05 \pm 0.30^{\mathrm{a}}$ & $-6.60 \pm 0.21$ & $-6.00 \pm 0.24$ & $-4.87 \pm 0.26^{\mathrm{a}, \mathrm{b}}$ \\
\hline
\end{tabular}

FBG, fasting blood glucose; Tch, total cholesterol; LDL-c, low-density lipoprotein cholesterol; TG, triglyceride; ISI, insulin sensitivity index; $\mathrm{DM}$, diabetes mellitus; Data are the means $\pm \mathrm{SD}$ values. ${ }^{\mathrm{a}} \mathrm{P}<0.05$ versus $\mathrm{DM}$ group. ${ }^{\mathrm{b}} \mathrm{P}<0.05 \mathrm{DM}+\operatorname{sitagliptin}(30 \mathrm{mg} / \mathrm{kg})$ group versus $\mathrm{DM}+\operatorname{sitagliptin}(10 \mathrm{mg} / \mathrm{kg})$ group.

A

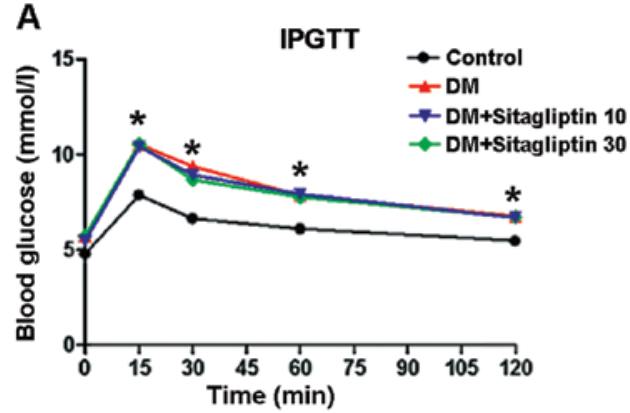

C

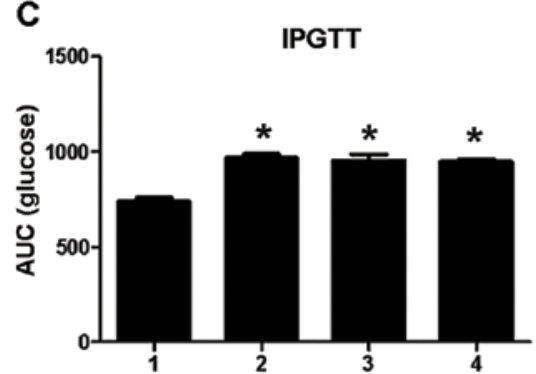

B

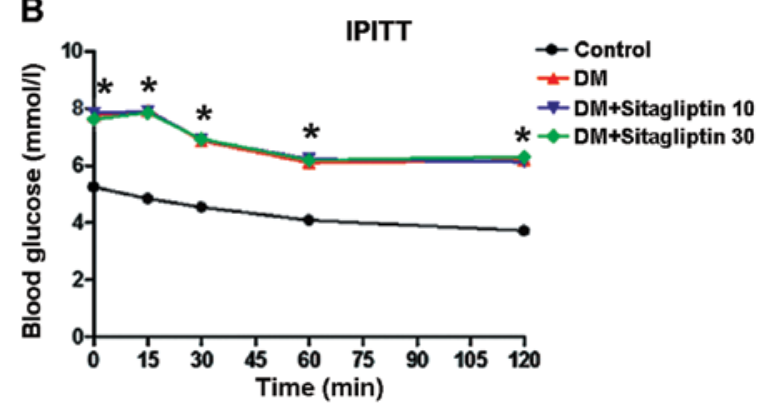

D

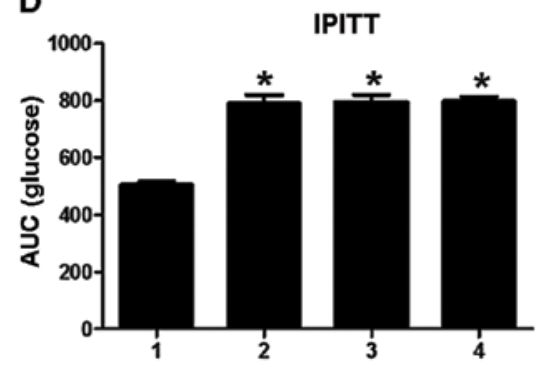

Figure 1. Intraperitoneal glucose tolerance test (IPGTT) and intraperitoneal insulin tolerance test (IPITT) results after feeding the rats a high-fat (HF) diet for 8 weeks. (A) Blood glucose levels during IPGTT. (B) Blood glucose levels during IPITT. (C) The area under the curve (AUC) of glucose in IPGTT. (D) The AUC of glucose in IPITT. Data are expressed as the means \pm standard deviation (SD). * $\mathrm{P}<0.05$ vs. control group. 1 , control group ( $\mathrm{n}=7$ ); 2 , diabetes mellitus (DM) group $(\mathrm{n}=6) ; 3, \mathrm{DM}+\operatorname{sitagliptin}(10 \mathrm{mg} / \mathrm{kg})$ group $(\mathrm{n}=6) ; 4, \mathrm{DM}+\operatorname{sitagliptin}(30 \mathrm{mg} / \mathrm{kg})$ group $(\mathrm{n}=6)$.
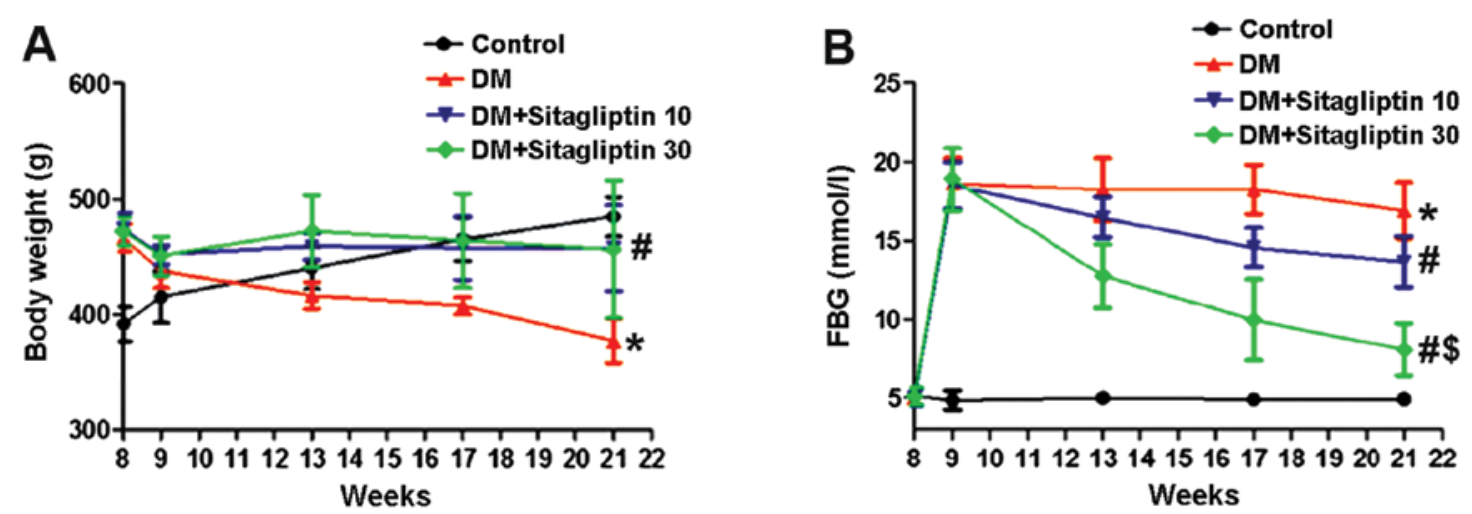

Figure 2. Dynamic variations in (A) body weight and (B) fasting blood glucose (FBG) during the treatment period. Data are expressed as the means \pm standard deviation (SD). ${ }^{*} \mathrm{P}<0.05$ diabetes mellitus (DM) group versus control group, ${ }^{\#} \mathrm{P}<0.05 \mathrm{DM}+$ sitagliptin group versus $\mathrm{DM}$ group, ${ }^{\$} \mathrm{P}<0.05 \mathrm{DM}+\mathrm{sitagliptin}(30 \mathrm{mg} / \mathrm{kg})$ group versus DM + sitagliptin $(10 \mathrm{mg} / \mathrm{kg})$ group. Control group $(\mathrm{n}=7) ; \mathrm{DM}$ group $(\mathrm{n}=6) ; \mathrm{DM}+\operatorname{sitagliptin}(10 \mathrm{mg} / \mathrm{kg}) \mathrm{group}(\mathrm{n}=6) ; \mathrm{DM}+\operatorname{sitagliptin}(30 \mathrm{mg} / \mathrm{kg})$ group $(\mathrm{n}=6)$. 

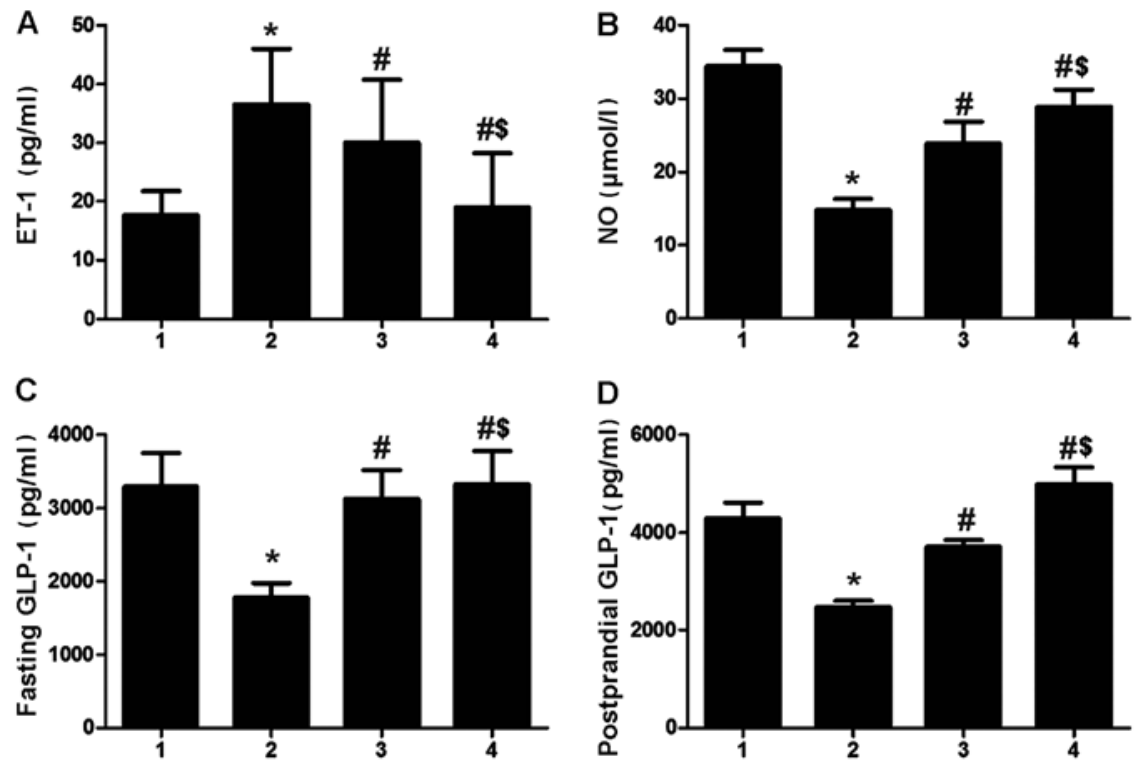

Figure 3. Serum levels of endothelin-1 (ET-1), nitric oxide(NO) and glucagon-like peptide-1 (GLP-1) following treatment with sitagliptin for 12 weeks. (A) ET-1 level. (B) NO level. (C) Fasting GLP-1 level. (D) Postprandial GLP-1 level. Data are expressed as the means \pm standard deviation (SD). "P<0.05 diabetes mellitus $(\mathrm{DM})$ group versus control group, ${ }^{\#} \mathrm{P}<0.05 \mathrm{DM}+$ sitagliptin group versus $\mathrm{DM}$ group, ${ }^{\mathrm{S}} \mathrm{P}<0.05 \mathrm{DM}+$ sitagliptin $(30 \mathrm{mg} / \mathrm{kg})$ group versus DM + sitagliptin $(10 \mathrm{mg} / \mathrm{kg}$ ) group. 1, Control group (n=7); 2, DM group (n=6); 3, DM+sitagliptin (10 mg/kg) group (n=6); 4, DM + sitagliptin (30 mg/kg) group (n=6).

A
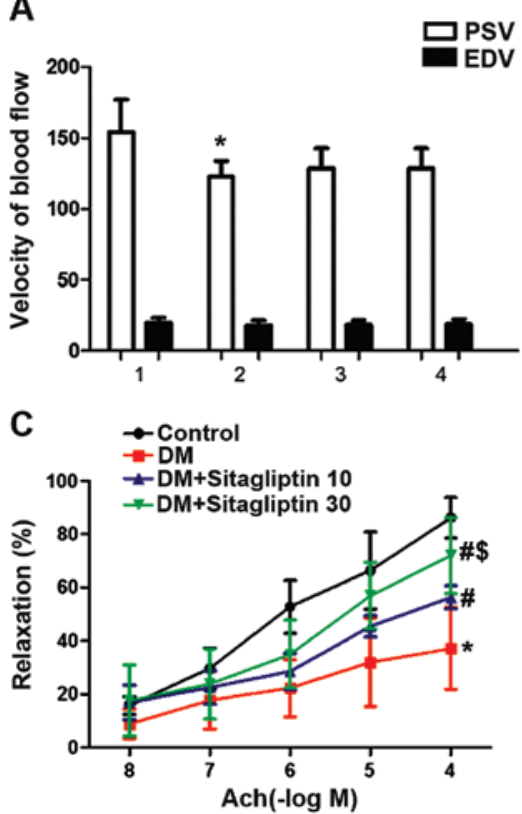

B

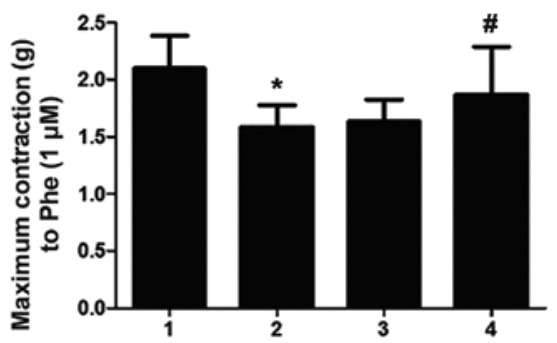

D

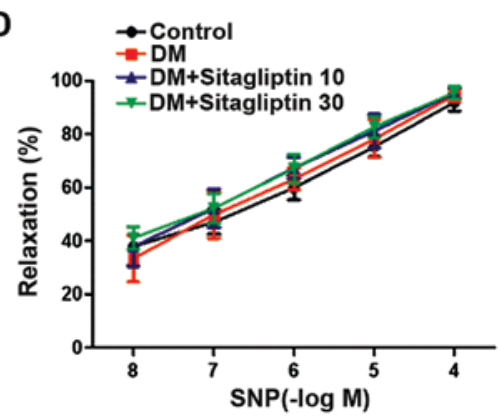

Figure 4. (A) Velocity of blood flow measured by non-invasive transcutaneous ultrasound. Maximum responses to (B) phenylephrine (Phe) as well as relaxation induced by (C) acetylcholine (Ach) and (D) sodium nitroprusside (SNP) in isolated rat thoracic aortic rings with or without therapy. Aortic rings were exposed to cumulative doses of the indicated agent. Values are presented as the percentage of relaxation relative to a Phe precontraction $(1 \mu \mathrm{M})$. Data are expressed as the means \pm (standard deviation) SD. ${ }^{*} \mathrm{P}<0.05$ diabetes mellitus (DM) group versus control group, ${ }^{\#} \mathrm{P}<0.05 \mathrm{DM}+$ sitagliptin group versus $\mathrm{DM}$ group, ${ }^{\$} \mathrm{P}<0.05$ $\mathrm{DM}+\operatorname{sitagliptin}(30 \mathrm{mg} / \mathrm{kg})$ group versus DM + sitagliptin $(10 \mathrm{mg} / \mathrm{kg})$ group. 1, control group $(\mathrm{n}=7) ; 2$, DM group (n=6); $3, \mathrm{DM}+\operatorname{sitagliptin}(10 \mathrm{mg} / \mathrm{kg})$ group $(\mathrm{n}=6) ; 4, \mathrm{DM}+$ sitagliptin $(30 \mathrm{mg} / \mathrm{kg})$ group $(\mathrm{n}=6)$. PSV, peak systolic velocity; EDV, end-diastolic velocity.

and was not significantly increased by the administration of sitagliptin $(\mathrm{P}<0.05)$ (Fig. 4A).

Morphological characteristics of rat aortic tissue. We examined the morphological characteristics of the arterial lesions in the H\&E-stained tissue sections. The endothelial cells adhering to inner elastic plates showed structural changes in the DM group compared with those in the control group.
Indeed, the vascular smooth muscle cells (VSMCs) of the tunica media were arranged irregularly, as shown by irregular nuclei and uneven staining of the cytoplasm, and the media layer thickness of the aorta was also markedly increased. However, the administration of sitagliptin attenuated the remodeling of the aortic intima, corrected the disarrangement of the VSMCs and reduced the media thickness of the aorta (Fig. 5A and C). 

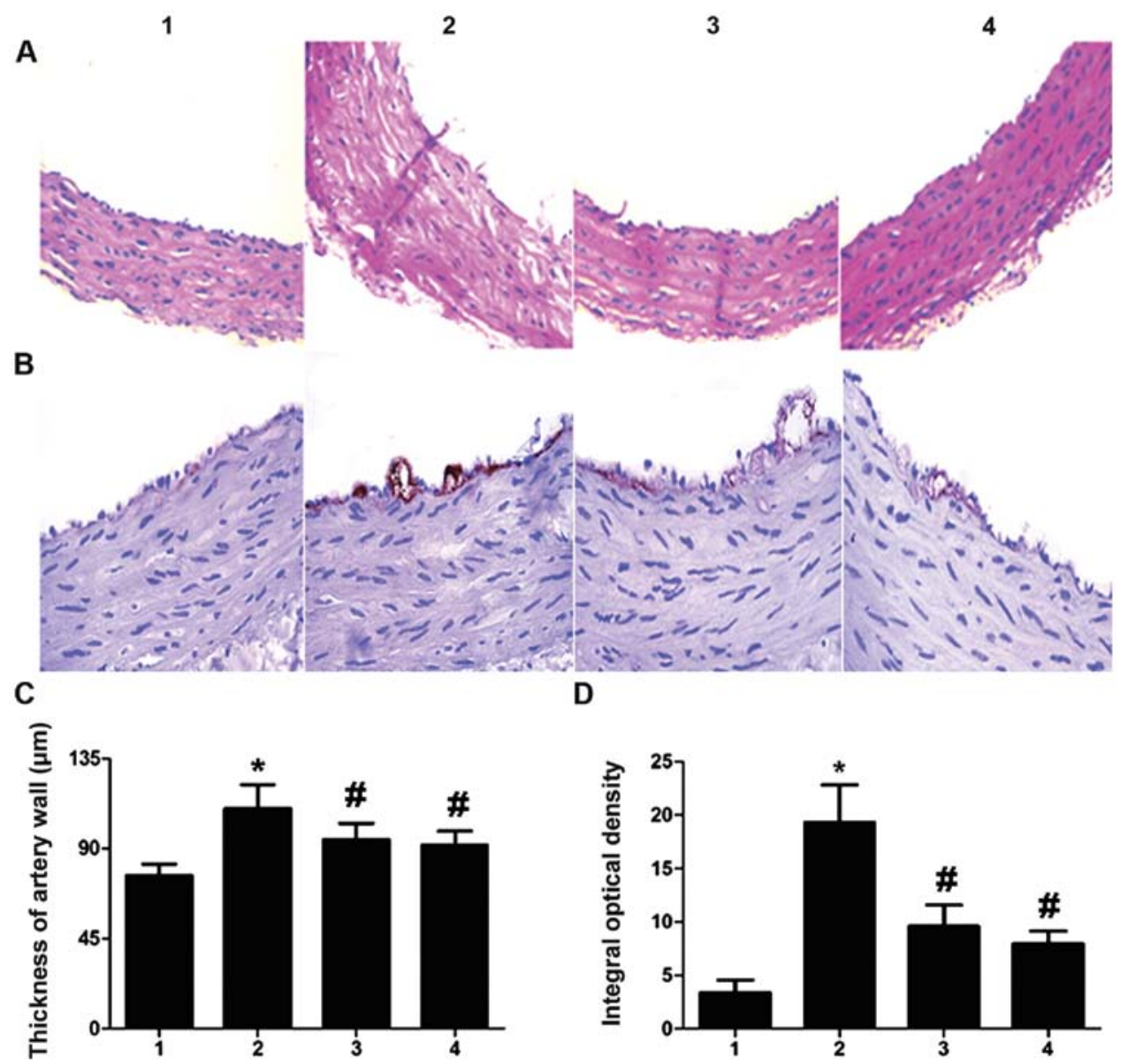

Figure 5. Sitagliptin attenuates aortic remodeling in diabetic rats and decreases endothelin-1 (ET-1) expression. (A) H\&E-stained sections of aorta (magnification, x200). (B) ET-1 protein expression was analyzed by immunohistochemical staining (magnification, x400). (C) Measurement of artery wall thickness. (D) Bar graph of protein quantification $(\mathrm{n}=6)$. Data are expressed as the means \pm standard deviation $(\mathrm{SD})$. ${ }^{*} \mathrm{P}<0.05$ diabetes mellitus (DM) group versus control group. ${ }^{\#} \mathrm{P}<0.05 \mathrm{DM}+$ sitagliptin group versus DM group. 1, control group (n=7); 2, DM group ( $\left.\mathrm{n}=6\right) ; 3$, DM + sitagliptin (10 mg/kg) group (n=6); 4, DM + sitagliptin $(30 \mathrm{mg} / \mathrm{kg})$ group $(\mathrm{n}=6)$.

As shown in Fig. 5B and D, a high ET-1 expression level was found in the intima of the DM rats, in comparison with that in the control rats $(\mathrm{P}<0.05)$. High- and low-dose administration of sitagliptin led to a significant downregulation of ET-1 expression in the intima of the arteries $(\mathrm{P}<0.05)$.

Relative gene and protein expression of signaling pathway components. As shown in Fig. 6A and B, hyperglycaemia and dyslipidaemia stimulated ET-1 expression at the transcriptional and translational levels, whereas the administration of sitagliptin significantly inhibited ET-1 expression $(\mathrm{P}<0.05)$. Western blot analysis also revealed that the rats in the DM group exhibited markedly enhanced expression of $\mathrm{p}-\mathrm{NF}-\mathrm{\kappa B}$, $\mathrm{NF}-\kappa \mathrm{B}$ and $\mathrm{p}-\mathrm{I} \kappa \mathrm{B} \alpha(\mathrm{P}<0.05)$, and reduced expression of p-AMPK as well as IкB $\alpha(\mathrm{P}<0.05)$ (Fig. 6C-E). Notably, the administration of sitagliptin reversed these effects on protein expression $(\mathrm{P}<0.05)$, thus, reversing the detrimental effects of hyperglycaemia and dyslipidaemia in the rat aorta.

\section{Discussion}

In the present study, we utilised streptozotocin injection to induce a defect in insulin secretion and a HF diet to induce an insulin-resistant state in a rat model of type 2 diabetes.
We have demonstrated that different doses of the DPP-4 inhibitor sitagliptin ameliorated glucose metabolism, insulin resistance and hemodynamic dysfunction to varying extents. Most importantly, sitagliptin markedly inhibited ET-1 expression at the transcriptional and translational levels, as well as ET-1-mediated endothelial dysfunction in the thoracic aorta samples isolated from rats with streptozotocin-induced diabetes. We suggest that the mechanism responsible for these effects potentially involves the activation of the AMPK signaling pathway through the elevation of GLP-1 levels, which subsequently suppresses the NF- $\kappa \mathrm{B} / \mathrm{I} \kappa \mathrm{B} \alpha$ system.

Several studies have indicated that DPP-4 inhibitors and GLP-1 analogues may exert beneficial effects on glucose metabolism and insulin resistance $(20,21)$. Our results showed that sitagliptin decreased FBG levels and alleviated insulin resistance in a dose-dependent manner, as GLP-1, which was elevated by the administration of sitagliptin to the diabetic rats, preserved plasma glucose homeostasis and protected $\beta$-cells in the pancreatic islets. Due to the enhancement of glucose metabolism, the weights of the rats in the treatment groups were also increased compared with the untreated rats. The lipid data were roughly similar to the findings of previous studies $(12,14)$, which reported that GLP-1 had minimal effects on the TG, Tch and LDL levels induced by a HF 


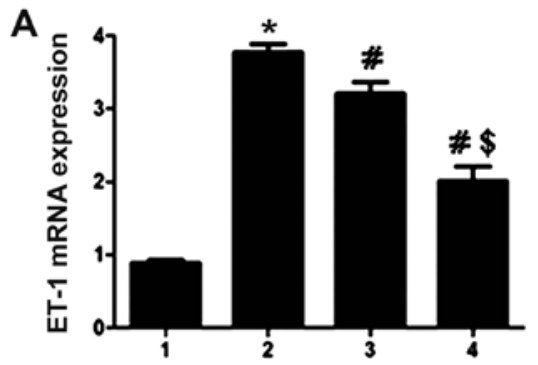

B
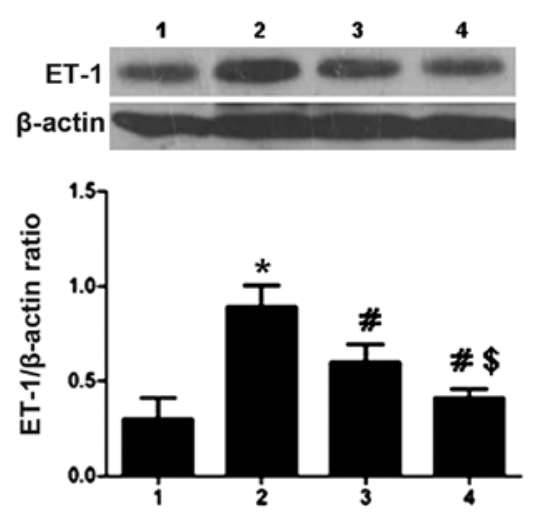

D
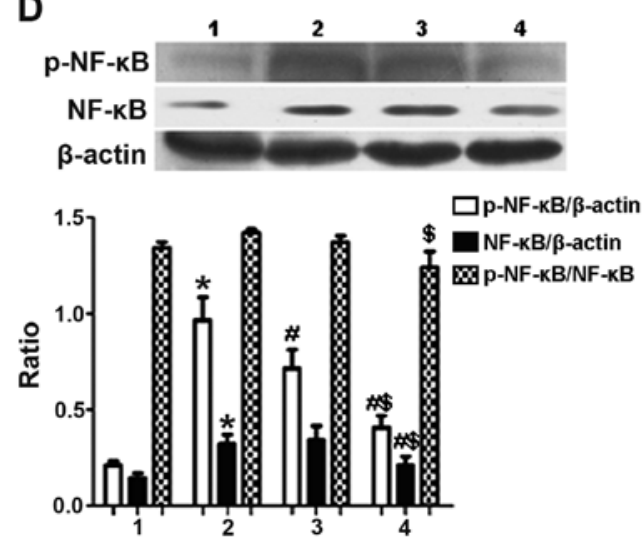

C
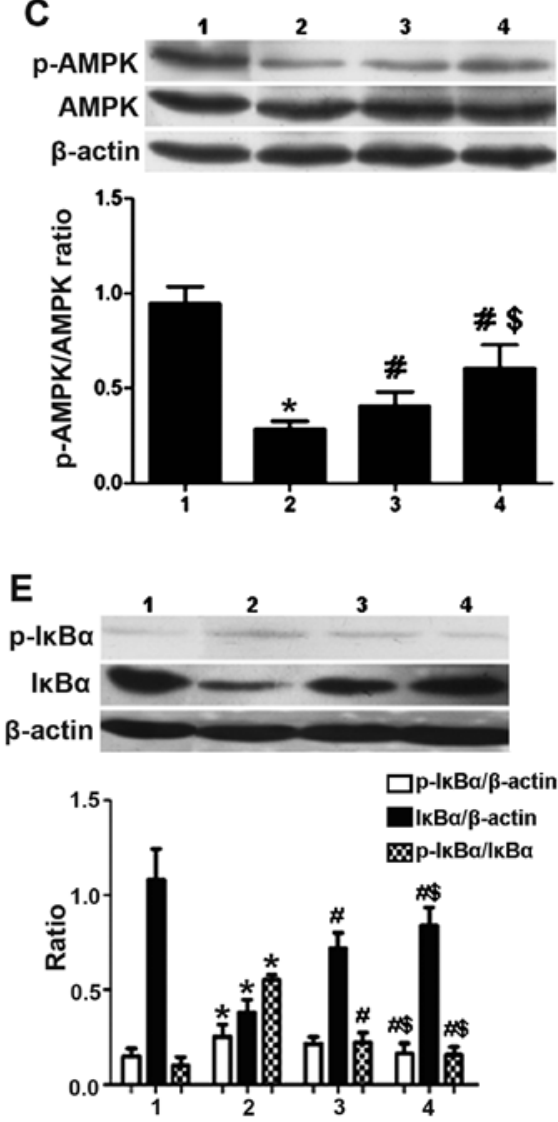

Figure 6. Effect of sitagliptin on (A and B) endothelin-1 (ET-1) expression as well as the phosphorylation of (C) AMP-activated protein kinase (AMPK), (D) nuclear factor $(\mathrm{NF})-\kappa \mathrm{B}$ and $(\mathrm{E}) \mathrm{I} \kappa \mathrm{B} \alpha$ in aortic samples isolated from diabetic rats. Data are expressed as the means \pm standard deviation (SD). $\mathrm{P}<0.05$ diabetes mellitus (DM) group versus control group, ${ }^{\#} \mathrm{P}<0.05 \mathrm{DM}+$ sitagliptin group versus $\mathrm{DM}$ group, ${ }^{\$} \mathrm{P}<0.05 \mathrm{DM}+$ sitagliptin $(30 \mathrm{mg} / \mathrm{kg})$ group versus $\mathrm{DM}+$ sitagliptin $(10 \mathrm{mg} / \mathrm{kg})$ group. 1, control group $(\mathrm{n}=7) ; 2$, DM group $(\mathrm{n}=6) ; 3, \mathrm{DM}+\operatorname{sitagliptin}(10 \mathrm{mg} / \mathrm{kg})$ group $(\mathrm{n}=6), 4, \mathrm{DM}+\operatorname{sitagliptin}(30 \mathrm{mg} / \mathrm{kg})$ group $(\mathrm{n}=6)$.

diet. The minimal changes in the lipid profiles following the administration of sitagliptin to diabetic rats, suggest that the vasoprotective and cardioprotective effects of sitagliptin may not be directly associated with its effects on lipid metabolism.

Emerging evidence $(4,22)$ has shown that diabetes, a coronary heart disease (CHD) equivalent, is closely associated with endothelial dysfunction, specifically with regard to the inability of the endothelium to regulate vascular homeostasis, which is a hallmark of vascular disease and atherosclerosis. The proposed mechanisms which are responsible for the endothelial damage occurring in diabetes, dependently and independently of the damage due to other cardiovascular risk factors, include hyperglycemia, insulin resistance, excessive free fatty acids (FFAs), oxidative stress and low-grade systemic inflammation $(4,5)$. Endothelial dysfunction is characterized by subdued vasodilation through reduced NO synthesis which induces platelet aggregation, smooth muscle cell proliferation and the nuclear transcription of leukocyte-adhesion molecules, as well as augmented vasoconstriction $(4,5)$. The endothelium secretes the potent vasoconstrictor ET-1 which promotes the development of cardiovascular disease through its pro-inflammatory and mitogenic effects (23). Current evidence links endothelial dysfunction with type 1 and type 2 diabetes, by demonstrating impaired endothelial-dependent vasorelaxation (4). Indeed, data from the present study has demonstrated that the serum NO levels were markedly reduced and the serum ET-1 levels were significantly increased in the diabetic rats. Particularly, diabetes promotes ET-1 expression in the aorta. Furthermore, aortic rings were used to prove that Ach-induced endothelium-dependent vasodilation was severely impaired in diabetes and the contractile response to Phe was also much smaller as previously reported (24), indicating that arterial remodeling in the diabetic rats caused decreased elasticity and compliance of the aorta as well as reduced endothelial function. 
As a member of a new class of anti-diabetic drugs, sitagliptin enhances glucose metabolism by increasing the active concentration and duration of action of GLP-1. It has been previously reported that GLP-1 reduces the progression of atherosclerosis by attenuating endothelial dysfunction (8-10,12-15) and we have further verified that sitagliptin enhances endothelial-dependent vasorelaxation in a dose-dependent manner. Previous studies have emphasized that GLP-1 stimulated NO production by enhancing the expression of eNOS $(14,15)$. In the present study, it is the first time to the best of our knowledge that a study has mainly focused on the effect of GLP-1 on ET-1 expression in diabetic rats. Firstly, the results revealed that sitagliptin markedly enhanced serum GLP-1 levels and inhibited serum ET-1 levels. Furthermore, as ET-1 expression is mainly modulated at a transcriptional level, we examined ET-1 expression at both the transcriptional and translational levels in the rat aortas, and the data showed that ET-1 expression was inhibited by sitagliptin, which partly accounted for the attenuation of endothelial dysfunction. These findings provide additonal evidence for the cardioprotective effect of GLP-1.

Previous investigations have identified the factors which regulate ET-1, which may be partly mediated by NF- $\mathrm{BB}(25,26)$. Elevated levels of advanced glycation end products (AGEs) have been demonstrated to induce ET-1 expression (26). In addition, high concentrations of glucose have been shown to induce NF- $\mathrm{B}$ activation in human umbilical vein endothelial cells (HUVECs) (27). Quehenberger et al (26) have found that the transient transfection of bovine aortic endothelial cells (BAECs) with plasmids overexpressing $\mathrm{I} \kappa \mathrm{B} \alpha$ led to a partial inhibition of AGE-mediated ET-1 expression, and transient transfection with plasmids overexpressing NF- $\kappa \mathrm{B}$ p50 and p 65 resulted in the induction of ET-1 expression, which suggested that AGE-mediated ET-1 induction is controlled by NF- $\mathrm{B}$. In animal models, Piechota and Goraca (25) have also demonstrated that NF- $\kappa \mathrm{B}$ inhibition plays a protective role in ET-1-induced oxidative lung injury, highlighting the important role of the NF- $\kappa \mathrm{B}$ pathway in the ET-1-induced overproduction of ROS. As a transcriptional regulator of $\mathrm{NF}-\kappa \mathrm{B}, \mathrm{I} \kappa \mathrm{B}$ kinase binds to and stabilizes $\mathrm{NF}-\kappa \mathrm{B}$. Following phosphorylation, I $\kappa \mathrm{B}$ kinase is degraded which results in the stimulation of NF- $\kappa \mathrm{B}$ phosphorylation (28). The involvement of the NF- $\kappa \mathrm{B} / \mathrm{I} \kappa \mathrm{B} / \mathrm{ET}-1$ pathway is consistent with our data regarding the enhancement of the phosphorylation of $\mathrm{I}_{\kappa} \mathrm{B} \alpha$ and $\mathrm{NF}-\kappa \mathrm{B}$, as well as the increased expression of the downstream target ET-1 in diabetic rat aortas.

It is well documented that exendin-4 inhibits monocyte adhesion to endothelial cells, and ameliorates atherosclerotic lesions in apolipoprotein E-deficient mice, by suppressing $\mathrm{NF}-\kappa \mathrm{B}$ activation in macrophages (29). Additionally, it has been previously reported that liraglutide exerted an anti-inflammatory effect on HUVECs by increasing NO generation and inhibiting NF- $\kappa \mathrm{B}$ activation, which was blocked by an AMPK inhibitor, suggesting that the action of liraglutide on NF- $\mathrm{NB}$ may occur partly through AMPK activation (30). In vivo, it has been reported that sitagliptin attenuates the progression of atherosclerosis in apolipoprotein-E knockout mice through AMPK-dependent mechanisms (31). Thus, we hypothesized that sitagliptin enhances serum GLP-1 levels, which may inhibit $\mathrm{NF}-\kappa \mathrm{B}$ activation by regulating the AMPK signaling pathway in the aorta. To verify this hypothesis in vivo, we established a rat model of streptozotocin-induced diabetes and administered sitagliptin for 12 weeks. The suppression of $\mathrm{I} \kappa \mathrm{B} \alpha$ and $\mathrm{NF}-\kappa \mathrm{B}$ phosphorylation as well as the activation of AMPK in rat aortas were observed in the high-dose sitagliptin group. Taking into account the finding that ET-1 expression is inhibited, we suggest that the downregulation of ET-1 expression by sitagliptin in the aortic endothelium is closely associated with the AMPK/NF- $\kappa \mathrm{B} /$ $\mathrm{I} \kappa \mathrm{B} \alpha$ signaling pathway. This conclusion is partly supported by a previous in vitro study, which demonstrated that liraglutide activated GLP-1 receptors and suppressed ET-1 expression in high glucose-cultured HUVECs by inhibiting NF- $\kappa \mathrm{B}$ phosphorylation and $\mathrm{NF}-\kappa \mathrm{B}$ translocation from the cytoplasm to the nucleus (32). However, the downregulation of ET-1 expression in the aortic endothelium may be associated with the decreased glucose levels in the treatment groups. However, an in vitro study by Dai et al (32) has noted that the effects of GLP-1 on ET-1 expression and upstream signaling pathways may occur independently of glucose metabolism, to a certain extent.

In summary, the present study has confirmed that sitagliptin apparently attenuates endothelial dysfunction and delays the progression of atherosclerosis in a dose-dependent manner in an animal model of diabetes. It is noteworthy that the findings of our study indicate that sitagliptin inhibits ET-1 expression in the aortic endothelium by suppressing the $\mathrm{NF}-\kappa \mathrm{B} / \mathrm{I} \kappa \mathrm{B} \alpha$ system through the activation of the AMPK pathway. By determining the effect of sitagliptin on ET-1 expression in vivo we have identified a novel mechanism which contributes to our current understanding of the way in which GLP-1 attenuates endothelial dysfunction.

\section{Acknowledgements}

The present study was funded by the National Natural Science Foundation of China (nos. 81470568, 81270372 and 81070232), the Anhui Provincial Natural Science Foundation (no. 1608085MH168) and the Research Project for Practice Development of National TCM Clinical Research Bases (no. JDZX2015133).

\section{References}

1. Wang J, Luben R, Khaw KT, Bingham S, Wareham NJ and Forouhi NG: Dietary energy density predicts the risk of clinically incident type 2 diabetes: the EPIC-Norfolk study. Diabetes Care 31: 2120-2125, 2008.

2. Wilmot EG, Edwardson CL, Achana FA, Davies MJ, Gorely T, Gray LJ, Khunti K, Yates T and Biddle SJ: Sedentary time in adults and the association with diabetes, cardiovascular disease and death: systematic review and meta-analysis. Diabetologia 55: 2895-2905, 2012.

3. Li J, Dong Y, Wu T and Tong N: Differences between western and Asian type 2 diabetes patients in the incidence of vascular complications and mortality: A systematic review of randomized controlled trials on lowering blood glucose. J Diabetes: Dec 11, 2015 (Epub ahead of print).

4. Sena CM, Pereira AM and Seiça R: Endothelial dysfunction - a major mediator of diabetic vascular disease. Biochim Biophys Acta 1832: 2216-2231, 2013.

5. Roberts AC and Porter KE: Cellular and molecular mechanisms of endothelial dysfunction in diabetes. Diab Vasc Dis Res 10: 472-482, 2013.

6. Drucker DJ: Glucagon-like peptides. Diabetes 47: 159-169, 1998.

7. Luft VC, Schmidt MI, Pankow JS, Hoogeveen RC, Couper D, Heiss G and Duncan BB; Atherosclerosis Risk in Communities Investigators: Dipeptidyl peptidase IV and incident diabetes: the Atherosclerosis Risk in Communities (ARIC) study. Diabetes Care 33: 1109-1011, 2010. 
8. Matheeussen V1, Waumans Y, Martinet W, Van Goethem S, Van der Veken P, Scharpé S, Augustyns K, De Meyer GR and De Meester I: Dipeptidyl peptidase in atherosclerosis: expression and role in macrophage differentiation, activation and apoptosis. Basic Res Cardiol 108: 350, 2013.

9. Ban K, Noyan-Ashraf MH, Hoefer J, Bolz SS, Drucker DJ and Husain M: Cardioprotective and vasodilatory actions of glucagon-like peptide 1 receptor are mediated through both glucagon-like peptide 1 receptor-dependent and -independent pathways. Circulation 117: 2340-2350, 2008.

10. Nyström T1, Gutniak MK, Zhang Q, Zhang F, Holst JJ, Ahrén B and Sjöholm A: Effects of glucagon-like peptide-1 on endothelial function in type 2 diabetes patients with stable coronary artery disease. Am J Physiol Endocrinol Metab 287: E1209-E1215, 2004

11. Noyan-Ashraf MH, Shikatani EA, Schuiki I, Mukovozov I, Wu J, Li RK, Volchuk A, Robinson LA, Billia F, Drucker DJ and Husain M: A glucagon-like peptide-1 analog reverses the molecular pathology and cardiac dysfunction of a mouse model of obesity. Circulation 127: 74-85, 2013

12. Vittone F, Liberman A, Vasic D, Ostertag R, Esser M, Walcher D, Ludwig A, Marx N and Burgmaier M: Sitagliptin reduces plaque macrophage content and stabilises arteriosclerotic lesions in Apoe $\left(^{-/}\right)$mice. Diabetologia 55: 2267-2275, 2012.

13. Shah Z1, Kampfrath T, Deiuliis JA, Zhong J, Pineda C, Ying Z, Xu X, Lu B, Moffatt-Bruce S, Durairaj R, et al: Long-term dipeptidyl-peptidase 4 inhibition reduces atherosclerosis and inflammation via effects on monocyte recruitment and chemotaxis. Circulation 124: 2338-2349, 2011.

14. Matsubara J, Sugiyama S, Sugamura K, Nakamura T, Fujiwara Y, Akiyama E, Kurokawa H, Nozaki T, Ohba K, Konishi M, et al: A dipeptidyl peptidase-4 inhibitor, des-fluoro-sitagliptin, improves endothelial function and reduces atherosclerotic lesion formation in apolipoprotein E-deficient mice. J Am Coll Cardiol 59: 265-276, 2012.

15. Han L, Yu Y, Sun X and Wang B: Exendin-4 directly improves endothelial dysfunction in isolated aortas from obese rats through the cAMP or AMPK-eNOS pathways. Diabetes Res Clin Pract 97: 453-460, 2012.

16. Chen YX, Wang XQ, Fu Y, Yao YJ, Kong MY, Nie RQ and Wang JF: Pivotal role of inflammation in vascular endothelia dysfunction of hyperlipidemic rabbit and effects by atorvastatin. Int J Cardiol 146: 140-144, 2011

17. Drolet MC, Plante E, Battistini B, Couet J and Arsenault M: Early endothelial dysfunction in cholesterol-fed rabbits: a non-invasive in vivo ultrasound study. Cardiovasc Ultrasound 2: 10, 2004.

18. Tang ST, Su H, Zhang Q, Tang HQ, Wang CJ, Zhou Q, Wei W, Zhu HQ and Wang Y: Melatonin attenuates aortic endothelia permeability and arteriosclerosis in streptozotocin-induced diabetic rats: possible role of MLCK- and MLCP-dependent MLC phosphorylation. J Cardiovasc Pharmacol Ther 21: 82-92, 2016.

19. Zhou B1, Pan Y, Hu Z, Wang X, Han J, Zhou Q, Zhai Z and Wang Y: All-trans-retinoic acid ameliorated high fat diet-induced atherosclerosis in rabbits by inhibiting platelet activation and inflammation. J Biomed Biotechnol 2012: 259693, 2012.
20. Picatoste B1, Ramírez E, Caro-Vadillo A, Iborra C, Ares-Carrasco S, Egido J, Tuñón J and Lorenzo O: Sitagliptin reduces cardiac apoptosis, hypertrophy and fibrosis primarily by insulin-dependent mechanisms in experimental type-II diabetes. Potential roles of GLP-1 isoforms. PLoS One 8: e78330, 2013.

21. Zhao HJ, Xu Q, Zhang GY, Liu Y, Yu T, Li HY, Yang LN, Wang HY and Xie LB: Therapeutic effects of GLP-1 analog on rats with experimental diabetes mellitus. J Jilin University 33 : 474-479, 2007.

22. Pasterkamp G: Methods of accelerated atherosclerosis in diabetic patients. Heart 99: 743-749, 2013.

23. Böhm F and Pernow J: The importance of endothelin-1 for vascular dysfunction in cardiovascular disease. Cardiovasc Res 76: 8-18, 2007.

24. Cicek FA, Kandilci HB and Turan B: Role of ROCK upregulation in endothelial and smooth muscle vascular functions in diabetic rat aorta. Cardiovasc Diabetol 12: 51, 2013.

25. Piechota A and Goraca A: Influence of nuclear factor- $\mathrm{B}$ B inhibition on endothelin-1 induced lung edema and oxidative stress in rats. J Physiol Pharmacol 62: 183-188, 2011.

26. Quehenberger P, Bierhaus A, Fasching P, Muellner C, Klevesath M, Hong M, Stier G, Sattler M, Schleicher E, Speiser W and Nawroth PP: Endothelin 1 transcription is controlled by nuclear factor-kappaB in AGE- stimulated cultured endothelial cells. Diabetes 49: 1561-1570, 2000.

27. Ho FM, Lin WW, Chen BC, Chao CM, Yang CR, Lin LY, Lai CC, Liu SH and Liau CS: High glucose-induced apoptosis in human vascular endothelial cells is mediated through NF-kappaB and c-Jun NH2-terminal kinase pathway and prevented by PI3K/Akt eNOS pathway. Cell Signal 18: 391-399, 2006.

28. Suzuki J, Ogawa M, Muto S, Itai A, Isobe M, Hirata $Y$ and Nagai R: Novel IkB kinase inhibitors for treatment of nuclear factor-kB-related diseases. Expert Opin Investig Drugs 20: 395-405, 2011

29. Arakawa M, Mita T, Azuma K, Ebato C, Goto H, Nomiyama T, Fujitani Y, Hirose T, Kawamori R and Watada H: Inhibition of monocyte adhesion to endothelial cells and attenuation of atherosclerotic lesion by a glucagon-like peptide-1 receptor agonist, exendin-4. Diabetes 59: 1030-1037, 2010.

30. Hattori Y, Jojima T, Tomizawa A, Satoh H, Hattori S, Kasai K and Hayashi T: A glucagon-like peptide-1 (GLP-1) analogue, liraglutide, upregulates nitric oxide production and exerts anti-inflammatory action in endothelial cells. Diabetologia 53: 2256-2563, 2010.

31. Zeng Y, Li C, Guan M, Zheng Z, Li J, Xu W, Wang L, He F and Xue Y: The DPP-4 inhibitor sitagliptin attenuates the progress of atherosclerosis in apolipoprotein-E-knockout mice via AMPK-and MAPK-dependent mechanisms. Cardiovasc Diabetol 13: 32, 2014

32. Dai Y, Mehta JL and Chen M: Glucagon-like peptide-1 receptor agonist liraglutide inhibits endothelin-1 in endothelial cell by repressing nuclear factor-kappa B activation. Cardiovasc Drugs Ther 27: 371-380, 2013. 